\title{
A SOFTWARE FOR SIMULATION OF EFFICIENCY OF HPGe DETECTORS
}

\author{
L. H. Khiem ${ }^{1}$, T. D. Trong ${ }^{2}$ \\ Institute of Physics, Vietnam Academy of Science and Technology, Hanoi \\ Joint Institute for Nuclear Research, Dubna
}

\begin{abstract}
Computer software named GE_EFF for calculation of detection efficiency of High Purity Ge (HPGe) detectors recently developed by us is presented. A Monte Carlo method has been used for simulation. The software has been written in Visual Basic language. The calculated efficiencies for our detectors are in agreement with the measured values using standard $\gamma$-ray sources. The software has been used at our laboratory of Institute of Physics (Vietnam) for gamma-radiation measurements.

Представлено недавно разработанное нами программное обеспечение GE_EFF для вычисления эффективности регистрации на HPGe-детекторах. Для моделирования использован метод МонтеКарло. Программное обеспечение написано на языке Visual Basic. Рассчитанная эффективность для нашего детектора согласована с измеренным значением с использованием стандартных источников гамма-излучения. Данное программное обеспечение было использовано для измерения гамма-излучения в лаборатории Института физики (Вьетнам).
\end{abstract}

PACS: $02.70 . \mathrm{Uu} ; 29.30 . \mathrm{Kv} ; 29.40 . \mathrm{Wk}$

\section{INTRODUCTION}

High purity germanium detectors HPGe nowadays have been widely used at different laboratories of application of nuclear radiation in Vietnam. One of the most important parameters is detection efficiency. Generally, detection efficiency of HPGe detectors is experimentally determined by using a set of $\gamma$-ray standard sources, their activities are precisely known and their energies cover all region of interest [1]. Furthermore, their geometrical shapes should be very similar to the shapes of the samples under measurement. Practically, these strict requirements are not always satisfied. Another way for overcoming the practical difficulties mentioned above is to calculate the efficiency of HPGe detectors. The Monte Carlo method has been usually used for this purpose [2,3]. The main advantage of the calculation method in comparison with the experimental one is its flexibility. It can be applied for any shape of the measured samples and for any energy under investigation.

There are some famous softwares, which can be used for determination of detection efficiencies of HPGe detector such as GEANT, MCNP, etc. [4,5]. However, these softwares

\footnotetext{
${ }^{1}$ E-mail: lhkhiem@iop.vast.ac.vn

${ }^{2}$ E-mail: tdtrong@iop.vast.ac.vn
} 
are not easy for use. This is a reason for a fact that besides these softwares, many specific ones have been developed at different laboratories for this purpose. At our Institute of Physics, there are several HPGe detectors. In this report, the software named GE_EFF for calculation of detection efficiency of HPGe detectors recently developed by us is presented. The software has been used at our Laboratory of Nuclear Physics for gamma-radiation measurements. This work is a part of a project named "Development of Computing Science for Specialized Fields Based on Resource Sharing High Performance Computing Center at VAST" recently created by Vietnam Academy of Science and Technology.

\section{SIMULATION METHOD}

The geometrical configuration of measurement considered in our simulation is schematically depicted in Fig. 1. An isotropic source has a disk shape with the diameter $D_{s}$ and the thickness $T_{s}$. The distance between the source and the detector is $H_{s}$. The parameters are designed as input parameters for the program presented in the Table. Only $\gamma$ rays with energy less than $2 \mathrm{MeV}$ are considered in our simulation. Therefore, only three processes of photoelectric absorption, Compton scattering and pair production were taken into account. Other effects are assumed to be insignificant and were neglected.

Our algorithm of simulation is very similar to that described by B. Grosswendt and E. Waibel in [6]. However, there are some differences in our calculation. Firstly, all secondary electrons produced in interaction of $\gamma$ rays with germanium inside the detector active volume are assumed to be fully stopped. Secondly, the cross sections for the three primary $\gamma$-ray interaction processes were calculated directly in our program using the formulae written in [1].

\section{RESULTS AND DISCUSSIONS}

The validity of the program was tested by comparing the efficiencies calculated by our program with those obtained experimentally for different measurement geometries. The measurements were performed with a p-type HPGe detector of model GC-2018 of ORTEC Company, which is located at our laboratory. The main parameters of configuration of the detector are listed in the Table. The experimental efficiencies were measured experimentally using the standard sources of ${ }^{133} \mathrm{Ba},{ }^{241} \mathrm{Am}$ and ${ }^{152} \mathrm{Eu}$ supported by IAEA. It is mentioned

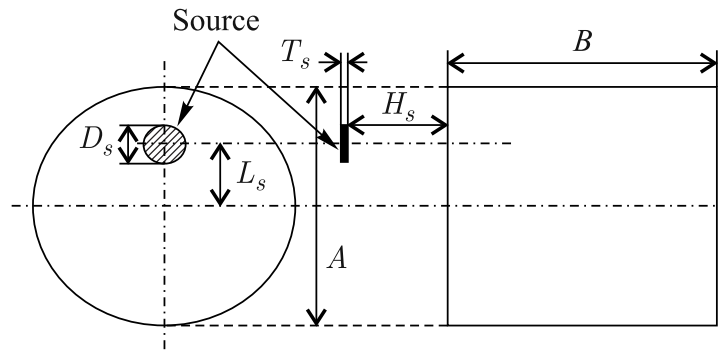

Fig. 1. The geometrical configuration of measurement used for simulation 
The main parameters of configuration of the detector HPGe model GC-2018 given by the manufacturer

\begin{tabular}{|l|c|}
\hline \multicolumn{1}{|c|}{ Detector parameter } & Value, $\mathrm{mm}$ \\
\hline Active Ge crystal diameter & 53.5 \\
Active Ge crystal length & 42.5 \\
Core diameter & 11 \\
Core depth & 31 \\
Radius of curvature of core & 5.5 \\
Aluminum/Mylar absorbed layer thickness & $0.03 / 0.003$ \\
Aluminum cover thickness & 1 \\
Distance from crystal to aluminum cover & 5 \\
Thickness of Ge/Li dead layer & 0.7 \\
Thickness of Ge/B dead layer & 0.0003 \\
\hline
\end{tabular}

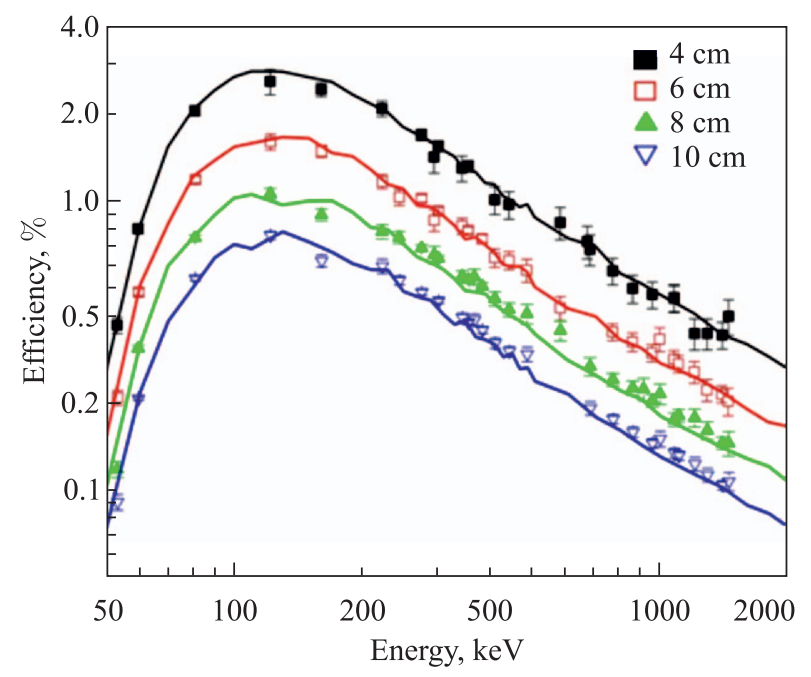

Fig. 2. The efficiencies calculated with GE_EFF using the new value of the thickness of the detector dead layer

that the thickness of dead layer in the Table is not true. We have measured it experimentally and a new value of $1.786 \mathrm{~mm}$ was found.

The efficiencies calculated for our detector using the GE_EFF program and the new value of the thickness of dead layer are plotted in Fig. 2. The calculation was done for a point source located at four distances away from the front face of the detector on its symmetrical axis. The calculated efficiencies were plotted in the same figure by solid lines. They are in very good agreement for a whole energy range from 60 to $1800 \mathrm{keV}$.

\section{CONCLUSIONS}

The efficiencies calculated by our GE_EFF program for our HPGe detector agreed very well in the range from $60 \mathrm{keV}$ to $2.0 \mathrm{MeV}$ with those measured experimentally. The program is a useful tool for efficiency calibration of $\gamma$-ray HPGe detectors. 
This work is supported by Vietnam Academy of Science and Technology under project named "Development of Computing Science for Specialized Fields Based on Resource Sharing High Performance Computing Center at VAST".

\section{REFERENCES}

1. William R. Leo. Techniques for Nuclear and Particle Physics Experiments - A How-to-Approach. Second Revised Ed. New York; Berlin; Heidelberg: Springer-Verlag, 1994.

2. Debertin K., Grosswendt B. // Nucl. Instr. Meth. 1982. V.203. P. 343-352.

3. Hardy J. C. et al. // Appl. Rad. Isotopes. 2002. V. 56. P. 65-69.

4. Hurtado S., Garcia-Leon M., Garcia-Tenorio R. // Nucl. Instr. Meth. A. 2004. V. 518. P. 764-774.

5. Salgado C. M., Conti C., Becker P. // Appl. Rad. Isotopes. 2006. V. 64. P. 700-705.

6. Grosswendt B., Waibel E. // Nucl. Instr. Meth. 1975. V.131. P.143-156.

7. Cornejo Diaz N., Jurado Vargas M. // Nucl. Instr. Meth. A. 2008. V. 586. P. 204-210. 